\title{
Relationship between flanker identifiability and compatibility effect
}

\author{
WOLFGANG SCHWARZ and AXEL MECKLINGER \\ Freie Universität Berlin, Berlin, Germany
}

\begin{abstract}
What is the relation between the identifiability of masked flankers and their ability to induce compatibility effects in a letter classification task? Using a within-subjects design $(n=8)$, we first determined identification performance for two flankers $(\mathrm{H}$ or $\mathrm{N})$ around an irrelevant target letter as a function of the time (stimulus onset asynchrony, or SOA) after which the flankers were masked. In a second condition, subjects classified the central letter of the same stimulus patterns irrespectively of the identity of the flankers. The compatibility effects increased with increasing identification performance as a function of SOA, and we found a significant compatibility effect even at an SOA at which the identifiability of the flankers did not differ significantly from zero. We discuss the statistical power of our design and an interpretation of our results in terms of a dissociation between perceptual processes and processes directly activating the motor system (direct parameter specification; cf. Neumann, 1990).
\end{abstract}

In their influential study on the effects of flanking letters on the time required to classify a target, B. A. Eriksen and C. W. Eriksen (1974) demonstrated strong effects of the compatibility of the flanking context. Reaction times (RTs) for target classification are slower and error rates are larger when the target is flanked by letters that call for the competing response (incompatible condition) than when the target is surrounded by identical letters (compatible condition). This difference in RT between the two conditions is called the compatibility effect (cf. B. A. Eriksen \& C. W. Eriksen, 1974; C. W. Eriksen, Pan, \& Botella, 1993). Typically, neutral flanking letters that do not call for an experimentally defined response yield intermediate RTs and error rates, and inhibitory and facilitating effects are often measured in relation to this baseline. Subsequently, these basic findings were replicated and generalized in various directions (for recent reviews, see Cohen \& Shoup, 1993; or Miller, 1991a). For example, C. W. Eriksen and Schulz (1979) demonstrated that the magnitude of the compatibility effect can be influenced by the size and the figure-ground contrast of the target and the flankers. Moreover, C. W. Eriksen and Schulz (1979) and Flowers and Wilcox (1982) varied the relative stimulus onset asynchrony (SOA) of the target to the flankers and thereby traced the temporal dynamics of the compatibility effect.

C. W. Eriksen and his colleagues interpreted the effects of compatibility as evidence for limitations in focusing attention to a particular target caused by both target and flanker stimuli activating the response associated

Address correspondence concerning this article to W. Schwarz, Freie Universität Berlin. Psychologisches Institut. Habelschwerdter Allee 45. D-14195 Berlin, Germany, or to A. Mecklinger, who is now at the Max-Planck-Institute of Cognitive Neuroscience, Inselstrasse 2226, 04103 Leipzig. Germany (e-mail: meckling.at.zedat.fu-berlin.de). with them. They assume a continuous flow of information from the module that analyzes the visual input to the response activation system where both correct and incorrect responses receive priming and compete with each other. As a consequence, correct responses are inhibited and delayed in conditions with incompatible flankers. Evidence for the parallel activation of correct and incorrect responses in trials with incompatible flankers comes from studies recording electromyographic responses (Coles, Gratton, Bashore, C. W. Eriksen, \& Donchin, 1985; C. W. Eriksen, Coles, Morris, \& O'Hara, 1985) and movement-related aspects of the event-related potential (Gratton, Coles, Sirevaag, C. W. Eriksen, \& Donchin, 1988). Further evidence for a response locus of the compatibility effect can be derived from the fact that flankers that do not activate a competing response have little effect on target RT.

Although it is well established that the flanker effects can be manipulated by the spatial target-flanker distance (Andersen, 1990; Yantis \& Johnston, 1990), the gradient of attention (LaBerge, 1994; LaBerge, Brown, Carter, Bash, \& Hartley, 1991), or appropriate SOA variations (C. W. Eriksen \& Schulz, 1979), little attention has so far been paid to the relation between the identifiability of the flankers and the magnitude of the compatibility effect. Two principal positions seem to be possible in the conceiving of this relation, which in turn embrace a continuum of intermediate viewpoints. One view holds that complete identification of the flankers is a necessary condition for any compatibility effect to occur (see Holender, 1986). A different view has been spelled out by Neumann, for example (1990; Neumann, Koch, Niepel, \& Tappe, 1992; for a related neurophysiological approach, see Goodale \& Milner, 1992). According to his conceptualization, there are two routes along which processing may proceed from sensory input to the motor 
system: either by building an explicit mental representation ("perception"), or by direct specification of the relevant motor response parameters. According to Neumann, these two routes may be dissociated under certain experimental conditions, such as, for example, in experiments on the Fehrer-Raab effect (Fehrer \& Raab, 1962; but see Bernstein, Amundson, \& Schurman, 1973), or in comparisons of onset latency and temporal order judgments (Neumann et al., 1992). That is, a dissociation may exist whenever a given stimulus activates action parameters that are not compatible with the mental representation of the stimulus. An extreme example of dissociation is represented in studies designed to demonstrate subliminal perceptual effects by means of visual backward masking (for a review, see Cheesman \& Merikle, 1984; Duncan, 1987; Holender, 1986; Merikle, 1982; Reingold \& Merikle, 1988). However, we consider subliminal perception to be only the most extreme case on a continuum between complete association and complete dissociation of mental representations and observable motor responses.

Our aim in the present study was to investigate the relation between flanker identification performance and compatibility effects. Specifically, we included conditions in which the flankers were masked with different SOAs, ranging from SOAs at which flankers could not reliably be identified to SOAs where identification performance was well above chance. Subsequently, we determined whether effects of compatibility occurred under exactly the same conditions. The examination of the processing of flankers that cannot be identified by themselves is an example of the dissociation paradigm discussed extensively by Merikle and his colleagues (e.g., Reingold \& Merikle, 1988). For the present purposes, this paradigm first requires reliable assessment of the identifiability of the flankers (cf. Macmillan, 1986; Merikle, 1982). Identification thresholds are often defined as the maximum stimulus-mask delay, which still yields chance identification. Accordingly, a common procedure to establish identification thresholds is to use backward masking paradigms in which the SOA between a flanker and the mask is systematically decreased until verbal report accuracy falls to a predetermined threshold level - for example, $25 \%$ correct under a symmetric four-alternative forced choice design (cf. Cheesman \& Merikle, 1984). Subsequently, in a test phase the task under consideration is carried out with the threshold SOA previously determined (see Holender, 1986, for a critical review of the basic logic underlying these designs). In the present experiment, the test phase consisted of a compatibility task with masked flankers.

Several authors have pointed out in detail various possible pitfalls of the dissociation paradigm. For example, Purcell, Stewart, and Stanovich (1983) have shown that differential states of light adaptation during the threshold determination and the test phase may bias the results, because flankers that are not identifiable during the threshold determination phase may become identifiable during the test phase. Given this objection, only those studies can be considered valid in which strictly comparable light adaptation conditions are employed during threshold determination and the test phase. In addition, Cheesman and Merikle (1984) suggested that it is preferable to use several levels of flanker-mask SOAs even in the test phase in order to trace the time course of the induced flanker effects more neatly and eventually to compare it with that in an unmasked flanker condition, for which the underlying processes are often better understood. Another potential confounding variable is poststimulus cuing (Bernstein, Bissonnette, Vyas, \& Barclay, 1989). Bernstein et al. showed that a masked word prime may become easier (harder) to identify if it is followed by a semantically related (unrelated) target word. Thus, in order to establish valid flanker identification thresholds, conditions need to be used in which the influence of the flanker on the target is identical to that in the conditions used during the test phase. Finally, Merikle (1982) and Macmillan (1986) have pointed out that procedures for determining identification thresholds are often too inaccurate for one to be able to draw firm conclusions. More specifically, they argue that procedures for establishing identification thresholds have ( 1 ) to rely on a sufficient number of trials for obtaining statistically reliable response probabilities, and (2) to control for response biases during discriminated verbal reports.

In the present study, we have tried to meet these suggestions by using a relatively large number of trials in order to establish reliable identification threshold estimates, and by separating contributions of bias and sensitivity by means of a signal detection analysis. In addition, we tried to estimate the statistical power of our design explicitly by means of a simulation procedure. Finally, we chose a design under which the physical stimulus events as seen on the monitor during the threshold phase were completely identical to the stimulus events during the test phase. This ensured that no confounding effects due to differential light adaptation or poststimulus cuing could be invoked to explain possible effects observed under the test phase.

\section{METHOD}

Eight subjects, A-H (4 male, 4 female, 19-32 years old), participated in both conditions ( 1 and 2) of the experiment. They were students from the Free University of Berlin. All subjects had normal or corrected-to-normal vision; they were paid at a rate of $12 \mathrm{DM}(\approx \$ 8)$ per hour. Each subject performed the following two conditions on 2 consecutive days: on one day, one session of an identification condition (1); and on the other day, one session of a masked compatibility condition (2). The order of the conditions was counterbalanced across subjects.

Condition 1 served to measure flanker identification performance. Each trial started with a fixation point, which disappeared after $210 \mathrm{msec}$. Then, $28 \mathrm{msec}$ later, a central letter (either $\mathrm{H}$ or $\mathrm{N}$ ) appeared centered at the position of the fixation point, and, simultaneously, two identical flankers (either $\mathrm{H}$ or $\mathrm{N}$ ) were presented $44^{\prime}$ (center-to-center distance) to the left and to the right of the position of the cent ral jetter. After $14,28,42$, or $56 \mathrm{msec}$, the 
flankers were masked by a slightly larger random dot pattern, which did not change across trials. The delay between the presentation of the flankers and the presentation of the mask will be called the SOA of that particular trial. The task of the subjects was to identify the flankers while maintaining fixation on the central position. The subjects had to make their decisions ("H" or " $N$ ") by pressing one of two buttons on an external response key device, which was placed in front of the subjects. The next trial started $2 \mathrm{sec}$ later. The letters were presented in black color on a gray background of an Atari sm 124 monitor. The presentation was synchronized with the vertical scan of the video refresh cycle $(70 \mathrm{~Hz})$. The size of the letters was $4.4 \times 4.4 \mathrm{~mm}$. They were viewed from a distance of approximately $70 \mathrm{~cm}$, yielding a visual angle of $22^{\prime}$.

Following an initial block of practice, there were 25 blocks of 2 warm-up trials and 32 effective trials each, which contained each of the four combinations of central and flanker letters under each of the four SOAs exactly two times in a random order, yielding a total of 800 effective trials: 50 per combination of central letter (2), flanker (2), and SOA (4). Between the blocks, feedback was given concerning the percentage of correct responses, and the subjects were allowed to rest as long as they wanted to. Each session of Condition 1 lasted about $60 \mathrm{~min}$.

In Condition 2, the compatibility effect was measured as a function of the SOA between the flankers and their mask. Stimulus events and stimulus timing as seen on the monitor were identical to those in Condition 1, except for the following two points: (1) a condition was added in which the letters $W$ were used as flankers; and (2) a condition with SOA $=0$ msec was added, in which the central letter $\mathrm{H}$ or $\mathrm{N}$ was presented simultaneously with the two masks of the flankers. The task of the subjects in Condition 2 was to classify the central letter (called the farget in Condition 2) as quickly and accurately as possible by pressing one of two buttons of the external response key device. The target and the two masks disappeared immediately after the response. The next trial started 2 sec later. After each block, feedback was given concerning the mean RT of the correct responses during the last block, and the subjects were allowed to rest as long as they wanted to. Excluding one block of initial practice, a single session of Condition 2 consisted of 30 blocks of two warm-up trials and 26 effective trials: 24 trials containing each of the six target - flanker combinations (HHH, WHW, NHN, NNN, WNW, HNH) for each of the four SOAs 14, 28, 42, and $56 \mathrm{msec}$ exactly one time, and two trials in which the two targets $\mathrm{H}$ and $\mathrm{N}$ were presented simultaneously with the masks ( $\mathrm{SOA}=0 \mathrm{msec}$ ). These 26 trials in each of the blocks were presented in a random order, resulting in a total of 780 effective trials in Condition 2. A single session of Condition 2 lasted about $60 \mathrm{~min}$.

\section{RESULTS}

\section{Condition 1: Identification Performance}

To assess flanker identification performance (flanker $\mathrm{H}$ vs. flanker $\mathrm{N}$ ) in Condition 1, separate values of $d^{\prime}$ (Macmillan \& Creelman, 1991, p. 9) were calculated for each of the 8 subjects, four SOAs, and two central letters. Each $d^{\prime}$ is derived from a single hit and a single false alarm probability based on $n=50$ trials each. The basic results of Condition 1, pooled across different central letters, are shown in Table 1.

As is apparent from the table (bottom row), the range of SOAs chosen was appropriate to cover the transition zone from the chance level $d^{\prime}=0$ to a performance level which deviates from pure guessing. More specifically, Table 1 shows that for the shortest SOAs identification performance remained extremely low, and that it
Table 1 $d^{\prime}$ for Each Subject (A-H) and Stimulus Onset Asynchrony (SOA), Averaged Over the Two Central Letters $H$ and N

\begin{tabular}{lrrrr} 
& \multicolumn{4}{c}{ SOA } \\
\cline { 2 - 5 } Subject & \multicolumn{1}{c}{14} & \multicolumn{1}{c}{28} & \multicolumn{1}{c}{4} & 56 \\
\hline A & -.29 & -.10 & .18 & .35 \\
B & .26 & .10 & .11 & .20 \\
C & .35 & .19 & .13 & .28 \\
D & -.14 & -.11 & .15 & .26 \\
E & .10 & -.30 & -.13 & .38 \\
F & .08 & -.07 & .07 & -.13 \\
G & .36 & -.34 & .16 & .70 \\
H & .12 & -.03 & .56 & .52 \\
Mean $d^{\prime}$ & .10 & -.08 & .15 & .32 \\
$\chi^{2}(16)$ & 22.78 & 14.10 & 26.05 & 44.38 \\
$p$ & .12 & .59 & .06 & $<.01$ \\
\hline
\end{tabular}

Note--SOA is given in milliseconds. The $\chi^{2}(16)$ values as derived from the Gourevitch and Galanter (1967) test are indicated in the nextto-last line. The last line gives the associated $p$ levels.

reached a value substantially different from zero only for $\mathrm{SOA}=56 \mathrm{msec}$.

These observations were confirmed statistically. At the initial stage of the analysis, identification performance was not pooled across different central letters in order to first test whether the identifiability of the flankers depends on identity of the central letters ( $\mathrm{H}$ vs. $\mathrm{N}$; see Bernstein et al., 1989). Toward this end, individual values of $d^{\prime}$ were converted to $z$ scores following the procedure described by Gourevitch and Galanter (1967; see Macmillan \& Creelman, 1991, p. 271). Subsequently, the $8 \times 8=64$ individual $z$ scores were subjected to an analysis of variance (ANOVA) with the factors of SOA (four levels) and central letter (two levels). The main effect of SOA was significant $[F(3,21)=$ $4.29, p=.016]$, but neither the central letter $[F(1,7)<1]$ nor its interaction with the SOA $[F(3,21)<1]$ showed a significant effect. It was thus decided to average the sensitivity scores $d^{\prime}$ of each subject across the two central letters (cf. Macmillan \& Creelman, 1991, p. 275). Next, we tested whether mean sensitivities (i.e., $d^{\prime}$ values) for a given SOA differed significantly from zero. For a single SOA, the significance of the mean sensitivities based on $n$ individual values of $d^{\prime}$ may be tested by converting first the individual $z$ scores derived from each $d^{\prime}$ (Gourevitch \& Galanter, 1967) into $\chi^{2}(1)$ values and then summing the values of $\chi^{2}(1)$ over the 8 subjects and the two central letters. This procedure yields a single $\chi^{2}(16)$ value, given that the hypothesis of $d^{\prime}=0$ is actually correct for a given SOA. These values together with the associated significance levels are given at the bottom of Table 1.

For SOA $=14 \mathrm{msec}$ and $\mathrm{SOA}=28 \mathrm{msec}$, mean sensitivity did not differ significantly from zero $\left[\chi^{2}(16)=\right.$ $22.78, p=.12$ for SOA $=14 \mathrm{msec}$, and $\chi^{2}(16)=14.10$, $p=.59$ for $\mathrm{SOA}=28 \mathrm{msec}$, a result which also holds true for either central letter individually. For SOA = $42 \mathrm{msec}$, mean sensitivity approached marginal significance $\left[\chi^{2}(16)=26.05, p=.06\right]$, while for SOA $=$ 
$56 \mathrm{msec}$ it differed significantly from zero $\left[\chi^{2}(16)=\right.$ $44.38, p<.01]$. It should also be noted that these results for mean sensitivity are confirmed by the individual subjects' data. For example, for the SOA $=28 \mathrm{msec}$ there was not a single estimate of $d^{\prime}$ (out of 16 individual cases) that differed significantly from zero $(\alpha=.05)$.

Response bias usually refers to the preference of a subject for one of the two available responses (e.g., the response " $\mathrm{H}$ "). In Condition 1 , however, a different kind of response bias is conceivable, which consists in a preference of the subjects to report the central letter presented together with the flankers. If indeed the central letter induces this type of responding, we should expect a positive bias toward the answer " $\mathrm{H}$ " in the identification of the flanker under the presentation of $\mathrm{HHH}$ versus NHN, and similarly a positive bias toward the answer " $N$ " in the identification of the flanker under the presentation of NNN versus HNH. To test this hypothesis, we calculated the signal detection response bias measure $c$ (Macmillan \& Creelman, 1991, p. 33) separately for each central letter, SOA, and subject. Overall, there was a very slight bias $(\bar{c}=.04)$ toward the response " $\mathrm{N}$," but this bias was, in fact, larger for the central letter $\mathrm{H}(\bar{c}=.09)$ than for the central letter $\mathrm{N}(\bar{c}=$ $-.01)$. The estimated bias parameters $c$ were subjected to an ANOVA with the factors of central letter ( $\mathrm{H}$ vs. $\mathrm{N}$ ) and SOA (four levels). Neither of these two factors nor the interaction turned out to be significant.

\section{Condition 2: Compatibility Effect With Masked Flankers}

Incorrect responses were excluded from the RT analysis. The overall error rate equaled $3.1 \%$. The number of errors did not differ across SOAs, but it increased for compatible, neutral, and incompatible flankers, respectively $\left[\chi^{2}(2)=6.25, p=.04\right]$. Also, errors were somewhat $(21 \mathrm{msec})$ faster on the average than correct responses.

The mean RTs averaged over the two target letters $\mathrm{H}$ and $\mathrm{N}$ for each of the five SOAs are displayed in Figure 1. Mean RTs increased with increasing SOA. Starting at $\mathrm{SOA}=28 \mathrm{msec}$, compatible target-flanker combinations yielded shorter mean RTs than did neutral combinations (i.e., flanker W), while mean RTs to incompatible combinations were larger than they were in the neutral condition. This finding holds for both target letters ( $\mathrm{H}$ and $\mathrm{N}$ ) individually.

These descriptive results were confirmed statistically. Each of the 8 subjects conveyed 24 mean correct RTs: one for each target (H vs. N), each compatibility condition (compatible, neutral, and incompatible flanker), and each of the four SOAs. These $8 \times 24=192$ mean correct RTs were subjected to an ANOVA with the factors of target (two levels), compatibility (three levels), and SOA (four levels). As for the central letter in Condition 1, targets ( $\mathrm{H}$ vs. $\mathrm{N}$ ) did not show a significant effect $[F(1,7)<1]$, while there were significant main effects of both compatibility $[F(2,14)=9.22, p<.01]$ and SOA $[F(3,21)=13.11, p<.01]$. There was also a significant interaction between compatibility and SOA $[F(6,42)=$

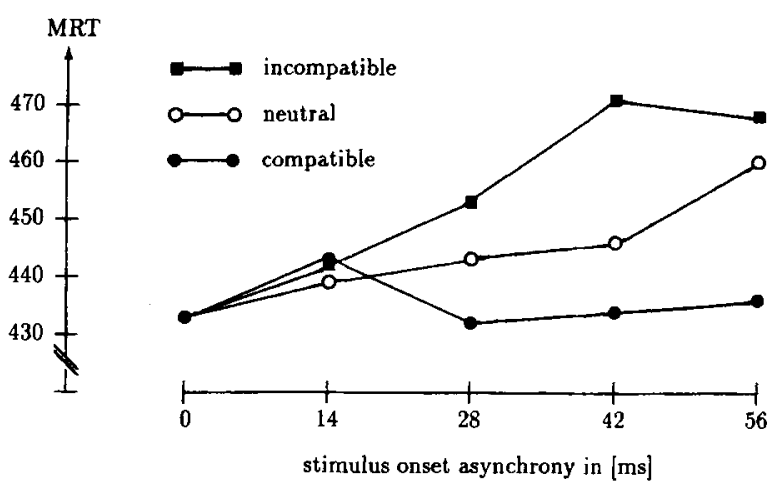

Figure 1. Mean correct reaction times (RTs; for 8 subjects) in Condition 2 as a function of stimulus onset asynchrony and targetflanker compatibility, averaged over both targets. Squares, incompatible flankers; open circles, neutral flankers; and closed circles, compatible flankers. $n \approx 465$ trials per point.

$4.30, p<.01]$, while all other effects were found to be insignificant $(\alpha=.05)$.

Figure 1 shows that with respect to the three compatibility conditions, speed and accuracy go in parallel, so that spurious correct "fast guesses" would, if anything, tend to favor the incompatible flanker condition. To ascertain which differences contributed to the significant overall effect of SOA, we compared mean RTs under different SOA compatibility combinations individually with Scheffe's post hoc test. For the SOAs of 28,42 , and $56 \mathrm{msec}$, the mean RTs of the compatible and the incompatible conditions differed significantly $(\alpha=.05)$, and these results held for each target letter separately. Specifically, for the SOA of $28 \mathrm{msec}$ the difference between the mean RTs from the compatible and the incompatible flanker condition equaled $453-432 \mathrm{msec}=21 \mathrm{msec}$, which exceeds the critical 5\% bound of Scheffe's test. For the two central letters, the mean RTs were $430 \mathrm{msec}$ for $\mathrm{HHH}$ versus $451 \mathrm{msec}$ for NHN, and $433 \mathrm{msec}$ for NNN versus $455 \mathrm{msec}$ for $\mathrm{HNH}$. Because this particular result for the SOA of $28 \mathrm{msec}$ was of some importance for our conclusions, we confirmed it nonparametrically by means of a simple sign test, which showed shorter mean RTs for the compatible than for incompatible flanker condition in 13 out of 16 comparisons for individual subjects $(p=.011)$.

\section{DISCUSSION}

The results reported in the present study indicate that the magnitude of the compatibility effect first reported by B. A. Eriksen and C. W. Eriksen (1974) gradually increases with the identifiability of the (masked) flankers. Significant compatibility effects were observed for flanker-mask delays of 28,42 , and $56 \mathrm{msec}$, while for an SOA of $14 \mathrm{msec}$, no reliable compatibility effects were obtained. Of particular importance is the pattern of results obtained for the $\mathrm{SOA}=28 \mathrm{msec}$ condition. While for the largest two SOAs the compatibility effects were paralleled by an above-chance performance for flanker 
identification (cf. Table 1), for SOA $=28 \mathrm{msec}$, a dissociation emerged. For this particular SOA, identification performance was not found to be significantly different from chance level, whereas a reliable compatibility effect was obtained under otherwise identical conditions.

Given that the displays used to measure identification performance and compatibility effects were physically identical, differential poststimulus cuing effects or differential light adaptation cannot explain the observed dissociation between identification performance and compatibility effects. However, identical stimulus displays are only a necessary, not a sufficient, condition for perceptually identical stimulation. One aspect of our experiments which was only indirectly controlled by instruction was the fixation of the eyes. We thus cannot definitively rule out that in Condition 1, contrary to the explicit instructions, the subjects directed their fixation to one of the positions where the flankers were known to be presented. What would be the probable effects on our data of fixating the flankers foveally? Given the inverse relation between retinal eccentricity and visual acuity (cf. Lie, 1980; Rijdijk, Kroon \& van der Wildt, 1980; Schwarz, 1993), such a behavior would clearly lead to an overestimation of the actual flanker identification performance under the conditions of the central letter classification task, where the targets are foveally fixated, and the flankers are fixated with a retinal eccentricity of $44^{\prime}$. Hence, the assumed foveal fixation of the flankers in Condition 1 would result in a conservative error, for it implies that under equal fixation conditions the compatibility effects would be even stronger than reported in the present study.

A simple interpretation of our data that needs to be evaluated is one in terms of practice effects that might have occurred during the course of the experiment (cf. Wolford, Marchak, \& Hughes, 1988). More specifically, suppose that successive presentations of the stimulus patterns during the flanker identification part of the experiment increased the ability of the subjects to identify the flankers. Given this assumption, it is conceivable that in a subsequent central letter classification task, flanker effects would have been due to their increased visibility. To evaluate this possibility, recall that 4 subjects performed the flanker identification condition first, whereas the other 4 subjects first classified the central letter. If there actually were practice effects of the sort just described, we should expect that the subjects who performed the flanker identification condition first would have exhibited stronger compatibility effects than did the subjects who started with the central letter classification task, because the former group had the opportunity to learn the flanker identification while the second did not.

However, several observations argue against a practice effect. First, for the three largest SOAs we found not even a descriptive advantage for the compatibility effect (the difference of mean RTs for incompatible vs. compatible flankers) of the subjects who identified the flankers first, while for the SOA of $14 \mathrm{msec}$ a difference of 4 msec emerged. Figure 2 shows the compatibility effects as a function of SOA, separately for each task order. On a descriptive level, these data do not support the notion that the compatibility effects observed in Condition 2 depended in any way on the order in which the subjects performed the tasks.

To confirm these results, we carried out a further ANOVA on the RT data of Condition 2, with task order as a between-subjects factor (two levels), and targetflanker compatibility (three levels) and SOA (four levels) as within-subjects factors. As might be expected from Figure 2, the results did not support the assumption of practice effects: neither the main effect of task order $[F(1,7)=1.08, p=.33]$ nor any of its interactions with the other factors proved to be significant. Second, if practice effects were present, it seems reasonable to expect the identification performance in Condition 1 would have increased during the course of the session. Accordingly, we inspected the data of the identification session by plotting the probability of a correct response as a function of the block number. There was no indication of a systematic trend related to the block number in these data. Comparisons of the performance in the first and the second half of the flanker identification experiment yielded a nonsignificant split of 5 subjects who improved and 3 subjects who did not. Finally, practice effects should, if actually present, also show up in the identification data, for the subjects who classified the central letter first (thereby possibly learning to identify the flanker) and then identified the flankers. Again, we found no indication of any systematic effect on identification performance related to task order. Specifically, the subjects who classified the central letter first did not subsequently identify the flankers any better than the subjects who started with the identification session. Of course, it is possible that the assumed practice effects might take some incubation period to show up, which would at least explain the absence of within-session

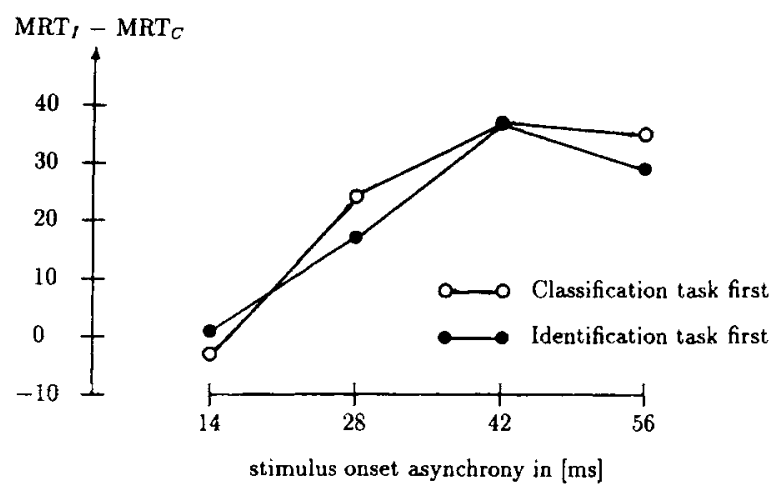

Figure 2. Net compatibility effect (mean reaction times, or RT, in incompatible trials minus mean $\mathrm{RT}$ in compatible trials) in Condition 2 as a function of stimulus onset asynchrony, separately for each task order. Closed circles refer to the 4 subjects who worked under Condition 1 (flanker identification) first, and then under Condition 2 (central letter classification). Open circles refer to the 4 subjects who performed the tasks in the opposite order. 
learning effects. It should be noted, however, that this assumption cannot explain the absence of task order effects.

Another simple interpretation of our results could be that there might have been a consistent ability to identify the flankers in Condition 1, even for an SOA of $28 \mathrm{msec}$, but that this ability was too weak to yield significant deviations from the hypothesis of $d^{\prime}=0$ (cf. Merikle, 1982; Miller, 1991b). Such a low identification performance in the compatibility experiment might, on the other hand, have been sufficient to induce small but reliable compatibility effects. To evaluate this alternative interpretation, it is crucial to estimate the statistical power of the identification Condition 1. Toward this end, the complete Condition 1 was simulated ten thousand times for a constant SOA. To simulate the performance of a given subject within a single trial, a normal distribution signal detection model with equal variances was assumed to hold with mean $d^{\prime}$ values (effect size) which varied from $d^{\prime}=0$ to $d^{\prime}=0.5$ in steps of 0.05 . Also, a slight subject variability around these mean $d^{\prime}$ values was introduced, which was chosen to match the variability estimated in Condition 1 . For each of the 8 "subjects" each simulation contained $n=50$ trials under each of the four target-flanker combinations, as in Condition 1. Two separate values of $d^{\prime}$ were estimated from the hit and false alarm rates for the pairs $\mathrm{HHH}$ versus NHN and NNN versus $\mathrm{HNH}$, which in turn were converted first to a $z$ score and then to a $X^{2}(1)$ value. Finally, the resulting 16 values of $\chi^{2}(1)$ (two values for each of 8 subjects) were pooled to obtain a single $\chi^{2}(16)$ value, which constituted the principal outcome from a single simulation run. From ten thousand replications of this procedure, we observed the relative frequency of cases in which a significant $(\alpha=.05)$ value of $\chi^{2}(16)>$ 26.30 was obtained. In other words, the power of the present study was estimated by simulation as a function of the given mean $d^{\prime}$ - that is, the effect size. The principal results of this procedure are shown in Figure 3.

From the results depicted in Figure 3, it is clear that for the number of subjects $(n=8)$ and trials $(n=50$ per combination of target, flanker, and SOA) employed in Condition 1, a reasonable power was obtained: for example, it is unlikely $(p=.04)$ that a mean sensitivity corresponding to $d^{\prime}=.35$ would have gone undetected. It is, on the other hand, equally clear that extremely low sensitivities such as, for example, $d^{\prime}=.10$ had only a rather low probability (e.g., $p=.13$ ) to be detected as a significant departure from the hypothesis $d^{\prime}=0$, and that the power necessarily converges to $\alpha=.05$ as mean $d^{\prime}$ approaches zero. Thus, one explanation of our results would be to assume a functional divergence between perceptual processes as revealed by identification performance and response activation processes in the sense that an extremely low degree of flanker identifiability may nevertheless lead to considerable compatibility effects as seen in the RT data of Condition 2.

An alternative interpretation of our findings is to assume a direct parameter specification of the motor sub-

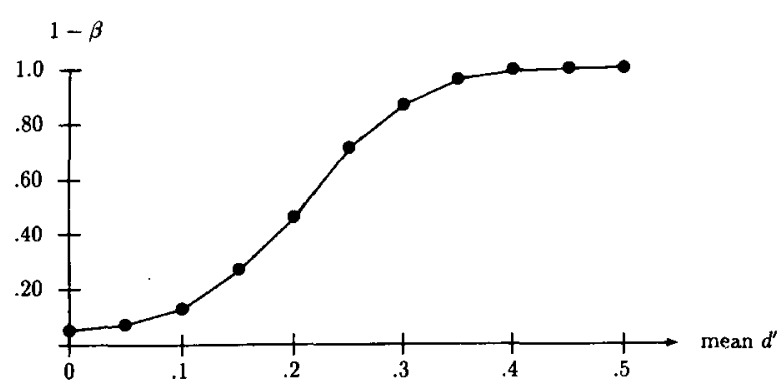

Figure 3. Probability $1-\beta$ to reject $\mathrm{H}_{0}: d^{\prime}=0$ under the design of Condition 1 of the present experiment as a function of the actual mean $d^{\prime}$ assumed in the simulation. $n=10,000$ simulation runs per estimated probability.

system associated with that particular flanker which is not tied to an explicit mental representation of the flanker ("dissociation," as discussed by Neumann, 1990; Neumann et al., 1992; or Goodale \& Milner, 1992). This activation may in turn induce response competition effects as typically observed with identifiable flankers. If this interpretation is correct, our data indicate that the dissociated activation of the motor subsystem by direct parameter specification is weaker than the response activation by explicit internal representation. It is conceivable that the larger compatibility effect for the larger flanker-mask SOAs $42 \mathrm{msec}$ and $56 \mathrm{msec}$ is attributable just to the additional contribution of the explicit mental representation to the total response activation. It should also be noted that in the present study, there was no preexperimental link between the stimuli (the letters $\mathrm{H}$ and $\mathrm{N})$ and the responses (left and right key). Under the interpretation of Neumann as described above, still larger effects might be expected if the stimuli are tied closer to the responses in advance of the experiment.

Two principal schemes are conceivable for making this reasoning more precise. On the basis of the notion of distinct discrete processing states (Jacoby, Toth, \& Yonelinas, 1993; Joordens \& Merikle, 1993; Roberts \& Sternberg, 1992), it may be assumed that the classification task studied in Condition 2 can be solved by using either of two processing modes. Under one processing mode, the flankers induce a direct specification of the associated response parameters only, whereas in the other processing mode, an explicit mental representation additionally coactivates the associated response. The probability of the two modes would then depend on the flanker-mask delay-the SOA. Under these assumptions, the increase of the magnitude of the compatibility effect with increasing flanker-mask delays could be attributed to an increasing proportion of trials under the second processing mode. On the other hand, even for short SOAs (e.g., $28 \mathrm{msec}$ ) that do not induce an explicit mental representation, a significant, though reduced, compatibility effect may be obtained in an increasing proportion of trials under the first processing mode. One problem in applying this model to the present experiments is that it is hard to separate the two contribu- 
tions-for example, by means of an explicit "inclusion" and "exclusion" instruction as proposed by Jacoby et al. (1993)-because of the unavoidable compatibility effects induced by the flankers.

An alternative concept that seems to fit the results of the present experiments better rests on the idea of continuous information processing (for reviews, see Miller, 1988, 1990; Ratcliff, 1988; Schwarz, 1989; or Schweickert, 1992, for a prototype of this class of models). This type of models assumes a continuous contribution to the total activation of each response alternative from both the target and the flanker. In compatible trials, both activations contribute to the same response alternative, and the related response activation may be assumed to be superposed (Schwarz, 1989). On the other hand, for incompatible trials, the contributions induced by the flankers and by the target cancel each other partially so that, in effect, inhibition results. For the present experiments, the contribution of the flanker to the total response activation is practically sure to have depended on the flanker-mask delay. More specifically, we assume that for short SOAs the contribution induced by the masked flanker may by itself have been too weak to activate the associated response (and to have been identified in Condition 1), but that it may nevertheless have summated with the larger activation induced by the target. The latter notion is consistent with the principle of subthreshold summation (both inhibitory and excitatory), which has long been known, for example, in visual psychophysics (cf. Graham, 1989; King-Smith \& Kulikowski, 1975, 1981). It is clear that a detailed test of these qualitative assumptions requires explicit quantitative formulation and parameteric experiments, which is beyond the scope of the present paper. If this "summation" interpretation of the present results is correct, they should probably not only obtain under a masking paradigm, but also under conditions that are more typical for subthreshold summation experiments, such as varying luminance or contrast of the flankers.

Collectively, the present results indicate that flankers that do not contain sufficient information to yield a correct identification response systematically affect the time to classify target stimuli. Although the power to detect above-chance identification performance was reasonably high, the possibility that the statistically reliable compatibility effects obtained for $\mathrm{SOA}=28 \mathrm{msec}$ were caused by extremely low degrees of flanker identifiability cannot unambigously be ruled out. However, the similarities in the magnitude of the compatibility effects and of identification performance as a function of SOA are consistent with models that assume a continuum between complete association and complete dissociation rather than a strict dichotomy between perceptual and motor activation processes.

Additional empirical evidence on the impact of degraded flankers on the classification of target letters is needed before a detailed model of these cognitive processes can be outlined. One useful strategy for future research to increase the power of the present approach might be to consider the relation of several dependent variables to the flanker-mask delay simultaneously. For example, it would be useful to consider the joint effects of compatibility on both speed and accuracy within the framework of a single explicit model (cf. Schwarz, 1990). Similarly, it would seem promising to attempt a combined analysis of performance and event-relatedpotential measures (cf. De Jong, Wierda, Mulder, \& Mulder, 1988; Gratton et al., 1988; Mecklinger, Kramer, \& Strayer, 1992) in order to examine dissociations and associations of perceptual and response activation processes in more detail.

\section{REFERENCES}

Andersen, G. J. (1990). Focused attention in three-dimensional space. Perception \& Psychophysics, 47, 112-120.

Bernstein, I. H., Amundson, V. E., \& Schurman, D. L. (1973). Metacontrast inferred from reaction time and verbal report: Replication and comment on the Fehrer-Biederman experiment. Journal of Experimental Psychology, 100, 195-201.

Bernstein, I. H., Bissonnette, V., Vyas, A., \& Barclay, P. (1989). Semantic priming: Subliminal perception or context? Perception \& Psychophysics, 45, 153-161.

Cheesman, J., \& Merikie, P. M. (1984). Priming with and without awareness. Perception \& Psychophysics, 36, 387-395.

Cohen, A., \& Shoup, R. E. (1993). Orientation asymmetry in the flanker task. Perception \& Psychophysics, 53, 693-703.

Coles, M. G. H., Gratton, G., Bashore, T. R., Eriksen, C. W., \& DonChIN, E. (1985). A psychophysiological investigation of the continuous flow model of human information processing. Journal of Experimental Psychology: Human Perception \& Performance, 11, 529-553

De Jong, R., Wierda, M., Mulder, G., \& Mulder, L. J. W. (1988). The timing of response preparation. Journal of Experimental $P_{S y-}$ chology: Human Perception \& Performance, 14, 682-692.

DUNCAN, J. (1987). Attention and reading: Wholes and parts in shape recognition--A tutorial review. In M. Coltheart (Ed.), Attention and performance XII (pp. 39-61). Hove, U.K.: Erlbaum.

ERIKSEN, B. A., \& ERIKSEN, C. W. (1974). Effects of noise letters upon the identification of a target letter in a nonsearch task. Perception \& Psychophysics, 16, 143-149.

Eriksen, C. W., Coles, M. G. H., Morris, L. R., \& O'Hara, W. P. (1985). An electromyographic examination of response competition. Bulletin of the Psychonomic Society, 23, 165-168.

Eriksen, C. W., Pan, K., \& Botella, J. (1993). Attentional distribution in visual space. Psychological Research, 56, 5-13.

ERIKSEN, C. W., \& SChulz, D. W. (1979). Information processing in visual search: A continuous flow conception and experimental results. Perception \& Psychophysics, 25, 249-263.

FEHRER, E., \& RAAB, E. (1962). Reaction to stimuli masked by metacontrast. Journal of Experimental Psychology, 63, 143-147.

FLOWERS, J. H., \& WiLCOX, N. (1982). The effect of flanking context on visual classification: The joint contribution of interactions at different processing levels. Perception \& Psychophysics, 32, 581-591.

GoOdAlE, M. A., \& MiLnER, D. A. (1992). Separate visual pathways for perception and action. Trends in Neurosciences, 15, 20-25.

Gourevitch, V., \& Galanter, E. (1967). A significance test for one parameter isosensitivity functions. Psychometrika, 32, 25-33.

Graham, N. V. S. (1989). Visual pattern analyzers. New York: Oxford University Press

Gratton, G., Coles, M. G. H., Sirevaag, E. J., Eriksen, C. W., \& Donchin, E. (1988). Pre- and post-stimulus activation of response channels: A psychophysiological analysis. Journal of Experimental Psychology: Human Perception \& Performance, 14, 331-344.

HoLENDER, D. (1986). Semantic activation without conseious perception in dichotic listening, parafoveal vision, and visual masking: A survey and appraisal. Behavioral \& Brain Sciences, 9, l-23.

JaCOBY, L. L., ToTH, J. P., \& Yonelinas, A. P. (1993). Separating con- 
scious and unconscious influences of memory: Measuring recollection. Journal of Experimental Psychology: General, 122, 139-154. JOORDENS, S., \& MERIKLE, P. M. (1993). Independence or redundancy? Two models of conscious and unconscious influences. Journal of Experimental Psychology: General, 122, 462-467.

King-Smith, P. E., \& KuLıKowski, J. J. (1975). The detection of gratings by independent activation of line detectors. Journal of Physiology, 247, 237-271.

King-Smith, P. E., \& Kulikowski, J. J. (1981). The detection and recognition of two lines. Vision Research, 21, 235-250.

LABERGE, D. (1994). Quantitative models of attention and response processes in shape identification tasks. Journal of Mathematical Psychology, 38, 198-243.

LaBerge, D., Brown, V., Carter, M., Bash, D., \& Hartley, A. (1991). Reducing the effects of adjacent distractors by narrowing attention. Journal of Experimental Psychology: Human Perception \& Performance, 17, 65-76.

LiE, I. (1980). Visual detection and resolution as a function of retinal locus. Vision Research, 20, 967-974.

MaCMillan, N. A. (1986). The psychophysics of subliminal perception. Behavioral \& Brain Sciences, 9, 38-39.

Macmillan, N. A., \& Creelman, C. D. (1991). Detection theory: A user's guide. Cambridge: Cambridge University Press.

Mecklinger, A., Kramer, A. F., \& Strayer, D. (1992). Event related potentials and EEG components in a semantic memory search task. Psychophysiology, 29, 104-119.

Merikie, P. M. (1982). Unconscious perception revisited. Perception \& Psychophysics, 31, 298-301.

Miller, J. [O.] (1988). Discrete and continuous models of human information processing: Theoretical distinctions and empirical results. Acta Psychologica, 67, 191-257.

MiLLER, J. O. (1990). Discreteness and continuity in models of human information processing. Acta Psychologica, 74, 297-318.

Miller, J. [O.] (1991a). The flanker compatibility effect as a function of visual angle, attentional focus, visual transients, and perceptual load: A search for boundary conditions. Perception \& Psychophysics, 49, 270-288.

MiLleR, J. [O.] (1991b). Threshold variability in subliminal perception experiments: Fixed threshold estimates reduce power to detect subliminal effects. Journal of Experimental Psychology: Human Perception \& Performance, 17, 841-851.

NeumanN, O. (1990). Direct parameter specification and the concept of perception. Psychological Research, 52, 207-215.
Neumann, O., Koch, R., Niepel, R., \& TAPPE, T. (1992). Reaktionszeit und zeitliches Reihenfolgeurteil: Übereinstimmung oder Dissoziation? Zeitschrift für experimentelle und angewandte Psychologie, 39, 621-645.

Purcell, D. G., Stewart, A. L.. \& Stanovich, K. E. (1983). Another look at semantic priming without awareness. Perception \& Psychophysics, 34, 65-71.

RATCLIFF, R. (1988). Continuous versus discrete information processing: Modeling accumulation of partial information. Psychological Review, 95, 238-255

Reingold, E. M., \& Merikle, P. M. (1988). Using direct and indirect measures to study perception without awareness. Perception \& Psy. chophysics, 44, 563-575.

RiJdiJ, J. P., Kroon, J. N., \& van der Wildt, G. J. (1980). Contrast sensitivity as a function of position on the retina. Vision Research, 20, 235-241.

Roberts, S., \& SternberG, S. (1992). The meaning of additive reaction-time effects: Tests of three alternatives. In D. E. Meyer \& S. Kornblum (Eds.), Attention and performance XIV (pp. 611-653). Cambridge, MA: MIT Press.

SCHWARZ, W. (1989). A new model to explain the redundant-signals effect. Perception \& Psychophysics, 46, 498-500.

SCHWARz, W. (1990). Stochastic accumulation of information in discrete time: Comparing exact results and Wald-approximations. Journal of Mathematical Psychology, 34, 229-236.

SCHWARz, W. (1993). A diffusion model of early visual search: Theoretical analysis and experimental results. Psychological Research. 55, 200-207.

SCHWEICKERT, R. (1992). Information, time, and the structure of mental events. In D. E. Meyer \& S. Kornblum (Eds.), Attention and performance XIV (pp. 535-566). Cambridge, MA: MIT Press.

Wolford, G., MarchaK, F., \& Hughes, H. (1988). Practice effects in backward masking. Journal of Experimental Psychology: Human Perception \& Performance, 14, 101-112.

YANTIS, S., \& JOHNSTON, J. C. (1990). On the locus of visual selection: Evidence from focused attention tasks. Journal of Experimental Psychology: Human Perception \& Performance, 16, 135-149.

(Manuscript received August 9, 1994; revision accepted for publication January $18,1995$. 